\title{
Rapid screening for bacteriuria using a particle counter, pulse-height analyser, and computer
}

\author{
MK ALEXANDER, MS KHAN, AND CS DOW
}

\begin{abstract}
From the Pathology Laboratory, Lakin Road, Warwick and Department of Biological Sciences, University of Warwick, Coventry CV4 7AL
\end{abstract}

SUMMARY A system for the rapid screening of urines for the presence of bacteriuria has been devised using a Coulter Counter Model ZBI linked to a multichannel pulse-height analyser (Coulter $\dot{\omega}_{+}$ 'Channelyser') with computer analysis of the output. In a series of 215 urines containing growth of aĩ single pathogen of more than $100 \times 10^{6} / 1(>100000 / \mathrm{ml})$ a satisfactory level of sensitivity $(99.1 \%) \overrightarrow{0}$ was obtained using only two different amplification settings by means of a brief treatment $\left(5-10_{0}^{+}\right.$ seconds) of the undiluted specimen with low intensity ultrasound; $85-90 \%$ of mixed growths of $10 \times 10^{6} / 1(>10000 / \mathrm{ml})$ were detected. Sonication did not improve the results in this group.T Specimens showing abnormal cyturia of more than $10 \times 10^{6} / 1(>10000 / \mathrm{ml})$ but no growth ono을 culture were positive in $33 \%$ of cases without the use of ultrasound but in $72 \%$ after sonication.

The examination of urine for the presence of bacterial infection forms a significant part of the workload of most hospital laboratories and is moreover a relatively lengthy procedure, entailing microscopy and the overnight incubation of cultures.

In our laboratory, which is probably representative, about $75 \%$ of examinations prove negative. A rapid and reliable method of screening should therefore have an appreciable impact on the work involved as well as on patient care.

Dow et al.,1 using a Coulter Counter Model 'ZBI' linked to a C 1000 multichannel pulse-height analyser, demonstrated that particle volume distribution analysis was capable of demonstrating bacteriuria with the required degree of sensitivity. We present the results of a further investigation of this approach using computer analysis of the analyser output as a first step in the production of an automated or semiautomated system. With this object in view and in the interests of speed of analysis, a procedure was sought that used an acceptably short counting time combined with the minimum number of different amplification settings.

\section{Material}

The material consisted of a fraction of the successive daily workload of the main and two peripheral

Accepted for publication 23 July 1980 laboratories. The selection of specimens was initially $\vec{\circ}$ random but biased in the latter part of both series (see below) by the deliberate selection of urines regarded as abnormal on microscopy. No selections was exercised in respect of age of specimen. Urine from a number of healthy subjects was also used inğ the establishment of normal patterns.

Routine bacteriological examinations were con $\overrightarrow{\overrightarrow{0}}$ ducted at each laboratory; in the peripheral labora -3 tories aliquots were formalinised by the addition of $10 \%$ formol-saline to a final concentration of $0.5 \%$. before they were transported. Other specimens. received by the main laboratory were similarlyo formalinised after routine sampling. In every case specimens were examined on the day of receipt. Two series were studied in succession, the second being? analysed after ultrasound treatment (see below).

\section{Methods}

BACTERIOLOGY

Cell counts were performed on well-mixed specimens using an improved Neubauer counting chamber. A centrifuged deposit was examined for casts, crystals and debris. Culture was carried out using a standar loop technique. ${ }^{2}$

The specimens were seeded on to cystine-lactose electrolyte deficient (CLED) agar (Diamed Diag? nostic) and incubated at $37^{\circ} \mathrm{C}$ overnight. For the detection of antibacterial substances a drop of urine was also placed on the surface of a Diagnostiọ Sensitivity test (DST) agar plate which had beers 194 
preinoculated with Escherichia coli NCTC 10418 and incubated in a similar manner. Plates were examined after 18-24 hours' incubation. Specimens showing no growth in the presence of pus cells were investigated for mycobacteria as well as for auxotrophs and anaerobic organisms. The bacteriological examinations were carried out by trained microbiology staff independently of the team (MKA and MSK) making the ZBI analysis, results being correlated retrospectively.

\section{ZBI ANALYSIS}

The apparatus used consisted of a Coulter Counter Model ZBI with a 30-micron diameter orifice tube linked to a C1000 Channelyser and thence by a standard Coulter interface to a teletype. The Channelyser is a pulse-height analyser which yields 100-channel histograms of particle volume distribution. The punched paper tape output was processed in a $32-\mathrm{K}$ word Nova $1200 \mathrm{mini}$ computer system. Instrument settings used were: aperture current 0.5 ; base channel threshold 15; window width 100 . Calibration was by standard latex particle suspensions. Urine was analysed at a dilution of 1 in 50 in Isoton using amplification settings of 2 and 8. In the second series, brief sonication (5-10 seconds) of $0.5 \mathrm{ml}$ of undiluted specimen was carried out before dilution using a small glassware-cleaning ultrasound bath $(50 / 60 \mathrm{kHz}, 80 \mathrm{~W})$.

\section{Interpretation of pulse height distributions}

The apparent volume distribution of a particle suspension as obtained by the Channelyser is affected by the simultaneous passage of two or more particles through the orifice of the counter and is a function of the true particle size distribution and concentration. Above an optimal particle concentration of $10-100 \times 10^{6} / 1$ a volume distribution peak becomes slurred and partly obliterated (Fig. 1).

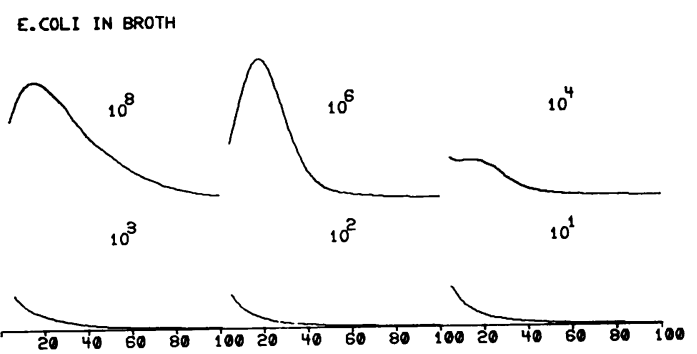

Fig. 1 Effect of different concentrations (per $\mathrm{ml}$ ) of an organism on the histogram of volume distribution. Amplification setting 2. X axes = analyser channel number; $Y$ axes $=$ number of pulses.

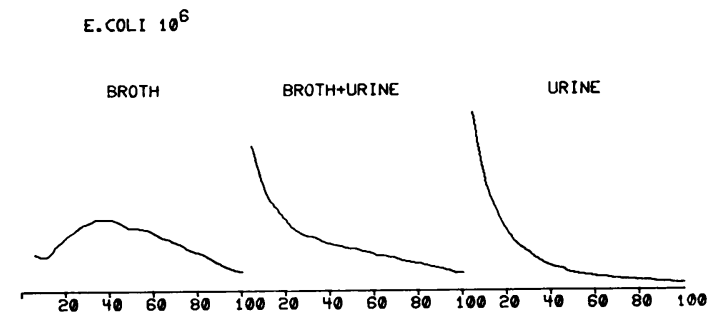

Fig. 2 Effect of urine 'background' particles on apparent volume distribution of $\mathrm{E}$. coli (overnight broth culture: final concentrations $100000 \times 10^{6} / \mathrm{l}$ in urine 1 in 50).

Apart from crystalline and other deposits, normal urine contains a population of particles of the order of 0.5 microns and below. These are believed to be the products of cytolysis of urinary tract epithelium ${ }^{3}$. The full characterisation of their volume distribution is beyond the sensitivity of the system used, the display at the highest amplifications normally showing a steep rise of pulse counts at the lower end of the volume range, although in specimens with unusually high particle concentrations a "coincidence peak' may appear. There is thus a natural background on which the changes due to the presence of bacteria are superimposed, and the pattern found in any particular specimen is a resultant of the concentration and volume distributions of background particles and any organisms present (Fig. 2).

\section{Principle of screening}

The method used consisted in the subtraction from each channel of each amplification the pulse count regarded as the maximum potentially attributable to background; if any channel count exceeded this maximum, the specimen was regarded as positive for bacteriuria. The maxima used were established from the examination of a series of normal urines, the criteria for normality being defined as a cell count by microscopy of less than $20 \times 10^{6} / 1(<20000 / \mathrm{ml})$, the absence of antibacterial substances, and the absence of growth on overnight incubation on CLED.

A 'worst case' histogram was constructed by computer analysis using the highest value found for each channel in the whole series of normals, the process being terminated when a number of further additions had failed to influence the results. Relative stability of the histograms was achieved from a series of about 50 urines.

\section{Results}

Specimens were classified in four groups on the basis of cell count and culture: those yielding a single 
Table 1 Results: First series (no ultrasound). Pure growths of a pathogen

\begin{tabular}{lccc}
\hline Organism $>10^{5} / \mathrm{ml}$ & No. & Positive & Negative \\
\hline E. coli & 64 & 59 & 5 \\
Klebsiella & 10 & 10 & - \\
Proteus & 13 & 12 & 1 \\
Pseudomonas & 3 & 3 & - \\
Staphylococcus & 6 & 4 & 1 \\
Str. faecalis & 5 & 4 & - \\
Other & 5 & 5 & 9 \\
Total & 106 & $97(91 \cdot 5 \%)$ & \\
\hline
\end{tabular}

organism in a concentration of greater than $100 \times$ $10^{6} / 1(>100000 / \mathrm{ml})$; mixed growths of more than $10 \times 10^{6} / 1(>10000 / \mathrm{ml})$; urines with a cell count of

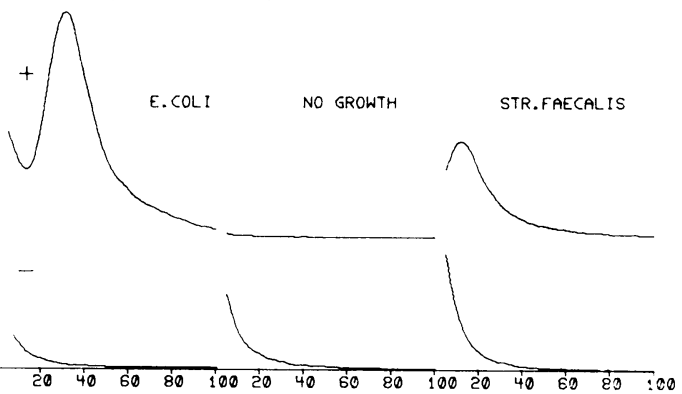

Fig. 3 Effect of ultrasound $(+)$ on histograms of 'background' particles and pathogens. more than $10 \times 10^{6} / 1(>10000 / \mathrm{ml})$ but with nథ growth on culture; and normals (as defined above) The results of the first series (Table 1) gave an overa detection rate of $91.5 \%$ for significant bacteriuriao the failure rate for Escherichia coli and staphyelococcus being particularly noteworthy.

It was at first thought that the failures might b $\mathrm{d}$ attributable to interference by high background counts, and a variety of methods were tried to diminish their effect. It was eventually found tha $\Phi$ a short period of treatment with ultrasound was indeed partially successful in removing background $\omega_{\sigma}$ In addition, however, it was discovered that in number of apparent negatives sonication resulted i⿱日 the sometimes dramatic appearance of peaks indicating significant bacteriuria (Fig. 3). Examin= ation of the state of suspensions of overnight brot? cultures (Fig. 4) showed that the effect of the lowe intensity ultrasound employed was to dispers bacterial aggregates. In this way clumps of organisms that would otherwise have been counted as sing large particles beyond the volume range of the amplifications used were separated into the individual components. Further evidence for this explanation is presented in the effect of increasing periods of ultrasound on the volume distribution of a suspension of staphylococcus (Fig. 5) where the

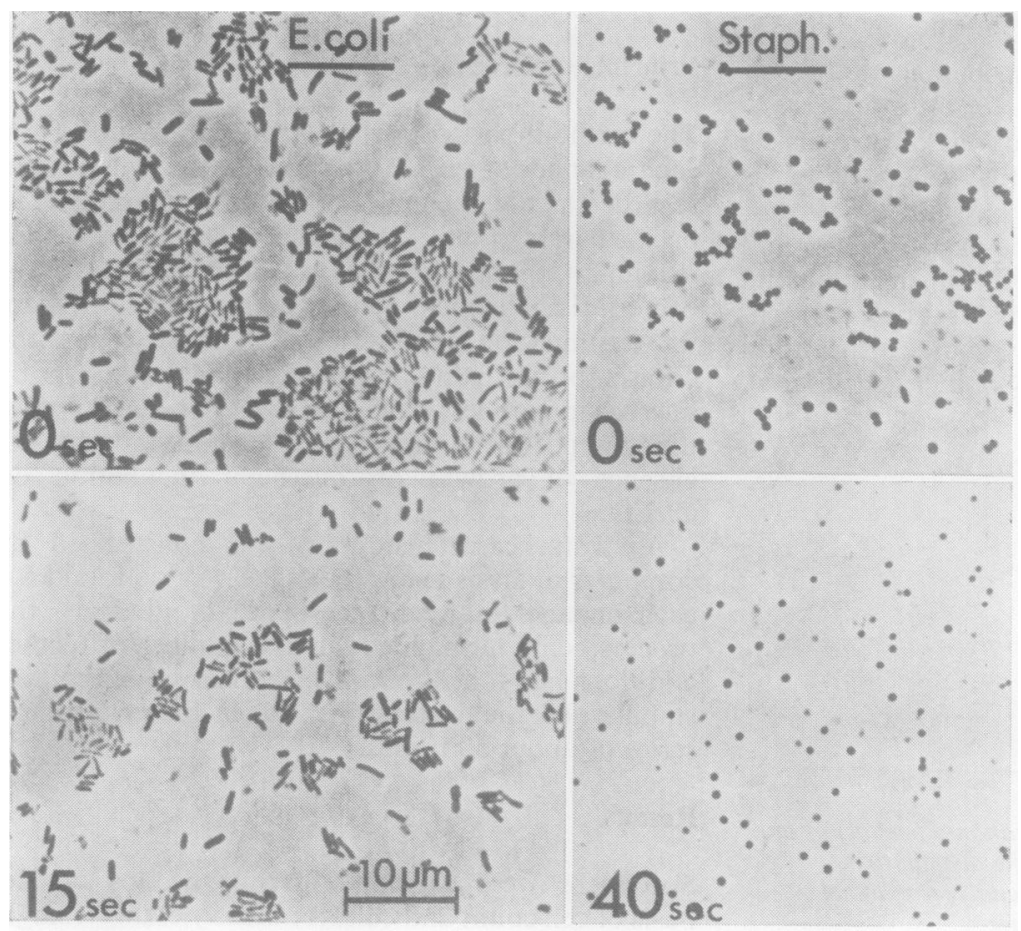

Fig. 4 Effect of brief sonications on suspensions of E. coli and staphylococcus. 


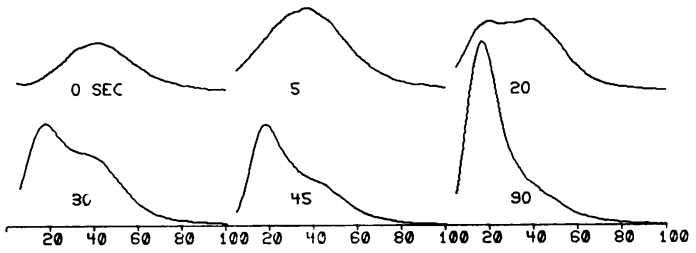

Fig. 5 Effect of increasing periods of sonication on the distribution histogram of a suspension of staphylococcus.

change first to a bimodal and subsequently to a sharp unimodal distribution may be seen.

Table 2 Results: Second series (pretreatment with ultrasound)

\begin{tabular}{lrcl}
\hline Organism $>10^{5} / \mathrm{ml}$ & No. & Positive & Negative \\
\hline E. coli & 133 & 133 & 0 \\
Klebsiella & 20 & 20 & 0 \\
Proteus & 21 & 20 & 1 \\
Pseudomonas & 7 & 7 & 0 \\
Staphylococcus & 13 & 13 & 0 \\
Staphylococcus & 13 & 13 & 0 \\
Str. faecalis & 10 & 10 & 0 \\
Other & 11 & 10 & 1 \\
Total & 215 & $213(99 \cdot 1 \%)$ & 2 \\
\hline
\end{tabular}

Table 3 Results: Mixed growths greater than $10 \times 10^{6} / l$ $(>10000 / \mathrm{ml})$

\begin{tabular}{llll}
\hline Ultrasound & No. & Positive & Negative \\
\hline Without & 21 & $19(91 \%)$ & 2 \\
With & 63 & $54(86 \%)$ & 9 \\
\hline
\end{tabular}

Table 4 Results: No growth. Cell count greater than $10 \times 10^{8} / \mathrm{l}(>10000 / \mathrm{ml})$

\begin{tabular}{llrl}
\hline Ultrasound & No. & Positive & Negative \\
\hline Without & 21 & $7(33 \%)$ & 14 \\
With & 76 & $55(72 \%)$ & 21 \\
\hline
\end{tabular}

A second series was therefore examined using pretreatment with ultrasound (see Methods) with improvement in sensitivity (Table 2). With two unexplained exceptions, the method now appeared to be fully sensitive for the detection of significant bacteriuria, notably all examples of $E$. coli and staphylococcus were now positive.

With regard to mixed growths (Table 3), the detection rate of $85-90 \%$ was not improved by the use of ultrasound; in contrast (Table 4), the $33 \%$ positive rate in cyturia without growth was increased to $72 \%$ by sonication.

\section{Discussion}

In order to translate the approach to screening for bacteriuria by particle volume distribution analysis into a practicable routine system it is necessary in the first place to substitute an objective and quantitative procedure for the qualitative method of assessment previously used ${ }^{1}$ and, in the second place, to shorten as far as possible the total time per analysis.

The restriction of the range of amplification settings used in the present study to two resulted in a loss of sensitivity compared to the wider range used in the original study as evidenced by the results for significant bacteriuria shown there when contrasted with those of the first (unsonicated) series in the present paper.

The difference may be attributed mainly to the omission of the lower amplifications in which alone any larger aggregates could be detected; their dispersion by ultrasound resulted in the component bacteria contributing to the patterns of the higher amplifications with recovery of the efficiency of the original method. The overall gain in analytical time by the present procedure is considerable, especially when combined with the shorter count time per amplification ( 30 as opposed to 60 seconds) and after allowance for the time used in sonication.

It would thus appear that with the inclusion of a preliminary treatment with low-intensity ultrasound, the system provides a highly sensitive and rapid method for the detection of significant bacteriuria. Specificity vis-à-vis normal urines is also satisfactorily high. The less than complete success in the detection of mixed growths of greater than $10 \times 10^{6} / 1$ $(>10000 / \mathrm{ml})$ may to some extent be a reflection of the limitations of the standard loop technique for assessing bacterial concentrations, a further possible explanation being the failure of a number of different species of differing mean volumes to summate sufficiently to produce a significant elevation of pulse heights at any one point. It is perhaps noteworthy that the specimens in this group that gave negative results by screening had all been reported as contaminant growths.

The findings in the group of specimens showing cyturia but no growth on culture are of particular interest. The exclusion from the series of specimens showing the presence of antibacterial substances and the culture methods employed make it unlikely that the findings could be the result of undetected bacteriuria, and the amplifications were too high to register the presence of intact cells. It therefore seems probable that the positive results in this group were due to the presence of cell stroma in excess of normal, a conclusion supported by the striking increase in positive findings produced by ultrasound. As the finding of a raised cell count is an essential part of screening for infection, the possibility of achieving this end by means of ultrasonic disintegration and detection of the resulting cellular 
debris seems to be a real one, and further experiments with varying periods of sonication are in progress.

Although, as previously demonstrated, ${ }^{1}$ under optimal conditions of growth and concentration an approach to taxonomic classification is possible, the present work shows this to be impracticable for routine urine specimens in view of the variations encountered in growth conditions and concentrations of organisms and background particles.

We thank the staff of the microbiology department of South Warwickshire District for their enthusiastic cooperation in this project.

\section{References}

${ }^{1}$ Dow CS, France AD, Khan MS, Johnson T. Particle size distribution analysis for the rapid detection of microbial蚂 infection of urine. J Clin Pathol 1979;32:386-90.

${ }^{2}$ O'Sullivan DJ, FitzGerald MG, Meynell MJ, Malins JM. A simplified method for the quantitative bacterial culture $\overline{\bar{N}}$ of urine. J Clin Pathol 1960;13:527-29.

${ }^{3}$ Smither R. Rapid screening for significant bacteriuria $\stackrel{\mathbb{D}}{\circ}$ using a Coulter Counter. J Clin Pathol 1977;30:158-62.

Requests for reprints to: Dr MK Alexander, Pathology $\vec{\circ}$ Laboratory, Lakin Road, Warwick. 